\title{
Mesial Rotation of the Upper First Molar: Association with Anterior Dental Crowding in Mixed and Permanent Dentition
}

\author{
Vincenzo Quinzi ${ }^{1,+} \oplus$, Simona Tecco ${ }^{2, *,+} \oplus$, Alessandro Nota ${ }^{2}{ }^{\circ}$, Eleonora Caggiati ${ }^{1}$, \\ Stefano Mummolo ${ }^{1}$ (D) and Giuseppe Marzo ${ }^{1}$ \\ 1 Deparment of Life, Health and Environmental Sciences, University of L'Aquila, 67100 L'Aquila, Italy; \\ vincenzo.quinzi@univaq.it (V.Q.); eleonora.caggiati@gmail.com (E.C.); stefano.mummolo@univaq.it (S.M.); \\ giuseppe.marzo@univaq.it (G.M.) \\ 2 Dental School, Vita-Salute San Raffaele University and IRCCS San Raffaele Hospital, 20132 Milano, Italy; \\ nota.alessandro@hsr.it \\ * Correspondence: tecco.simona@hsr.it \\ + The author were Principal Investigators with equal contribution.
}

Received: 5 July 2020; Accepted: 29 July 2020; Published: 31 July 2020

\begin{abstract}
The position of the upper first molar (UFM) is currently considered the "key of occlusion". The purpose of this study was to evaluate the prevalence of the mesiorotated UFM, and its relationship with anterior dental crowding in mixed and permanent dentition. Intra-oral scans of upper dental arches of 180 subjects with mixed dentition and 169 subjects with permanent dentition were retrospectively analyzed to investigate the presence of UFM mesiopalatal rotation and presence of anterior crowding. The occlusal relationship and the presence of caries were also recorded. In subjects with permanent dentition, there was a statistically significant correlation between anterior crowding and UFM mesiopalatal rotation (Pearson's chi-square $=9.03 ; p=0.06$ ). Among cases with permanent dentition, subjects with UFM mesiopalatal rotation showed an OR $=3.28(95 \%$ CI $=0.99-10.93$; $p=0.042$ ) of moderate/severe anterior dental crowding, respect to subjects without UFM rotation. In subjects with mixed dentition, there was a statistically significant correlation between molar occlusal relationship and UFM mesiopalatal rotation (Pearson's chi-square $=14.98 ; p=0.002$ ), and subjects with molar class II showed a OR $=10.2(95 \% \mathrm{CI}=2.16-48.22 ; p=0.0005)$ to present UFM mesiopalatal rotation, with respect to subjects with molar class I. UFM mesiopalatal rotation is associated to anterior dental crowding in permanent dentition, and to class II malocclusion in mixed dentition.
\end{abstract}

Keywords: dentistry; molar; dental occlusion; dental crowding; molar class; dental models; dental arch; interceptive orthodontics; orthodontics

\section{Introduction}

In 1899, Angle firstly described the importance of the position of the upper first permanent molar (UFM) in the diagnosis and planning of the orthodontic treatment. Thus, the UFM is considered the "key of occlusion" because of its stable anatomical position in relationship with the skull base, and its normal position [1-4] with the mesiobuccal cusp occluding in the buccal groove of the mandibular first molar.

Among the possible alterations of the UFM position, the occurrence of a mesiopalatal rotation in the previous literature seems to reach a prevalence of $83 \%$ in a population of subjects with presence of malocclusion [5-7], or even higher in subjects with class II malocclusion where it ranges from $83 \%$ to $95 \%$ [5,8-10]. Some authors hypothesized that the rotation itself could influence the correct occlusion with the lower first molar, generating a class II malocclusion [8,9]. UFM rotation has a 
considerable clinical relevance because it is able to influence three major aspects of the orthodontic treatment, as the molar relation in the sagittal plane [6,11], the available space in arch perimeter [12-17], and the long-term stability of orthodontic treatment outcomes related with better interdental contact points [18-20].

As there are few studies in literature evaluating the prevalence of UFM mesiopalatal rotation $[1,5,6,8,9,11,12,21]$, its relationship with dental occlusion was not as fully analyzed as, for example, the relationship with dental crowding and molar class relationship. Furthermore, most of the studies analyzed the prevalence of the UFM mesiopalatal rotation only in permanent dentition.

The aim of the present study is to analyze the association of mesiopalatal UFM rotation with anterior dental crowding in a sample of subjects with mixed or permanent dentition.

\section{Materials and Methods}

A retrospective study was carried out on a total sample of 349 subjects aged between 6 and 35 years (mean age $15.51+/-9.18$ ) whose digital intra-oral scans were obtained from two private dental clinics in Rome, Italy. One-hundred-and-eighty cases ( 90 males and 90 females, age range: 6-13 years, mean age: $8.42+/-1.83$ years) showed a mixed dentition, while 169 cases (64 males and 105 females, age range: 10-35 years, mean age: $22.67+/-8.02$ years) showed a permanent dentition. Inclusion criteria were presence of a fully erupted UFM; absence of significant dental anomalies in shape, size, and number; absence of fillings on UFM; no previous orthodontic treatment; availability of an orthopantomography; and availability of a signed informed consent form for treating patients data for research purposes. Furthermore, for subjects with mixed dentition, it the presence of the upper second primary molar was required.

The study was in accordance with the declaration of Helsinki and was approved by the ethical Committee of the University of L'Aquila (project code:2019/08, Document DR206/2013 of 08/02/2013).

In both groups, the UFM mesiopalatal rotation was calculated following the original Ricketts method [22] (Figure 1a,b): the line traced from the distobuccal cusp to the palatal cusp of the UFM (UFM line); it is considered ideal when it passes through the tip of the cusp of the contralateral canine (point $\mathrm{C}$ ). The smaller the distance between the line and the tip of the cusp of the contralateral canine (C), the better the rotational position of UFM; the molar is considered well positioned at distances lower than $4 \mathrm{~mm}$.

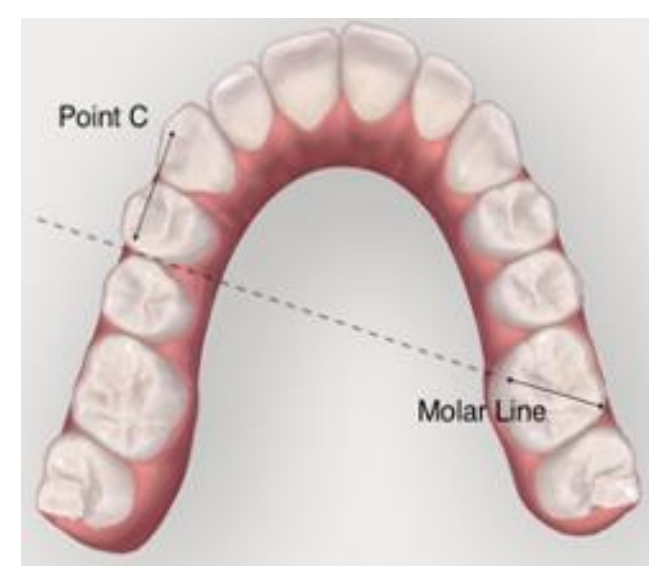

(a)

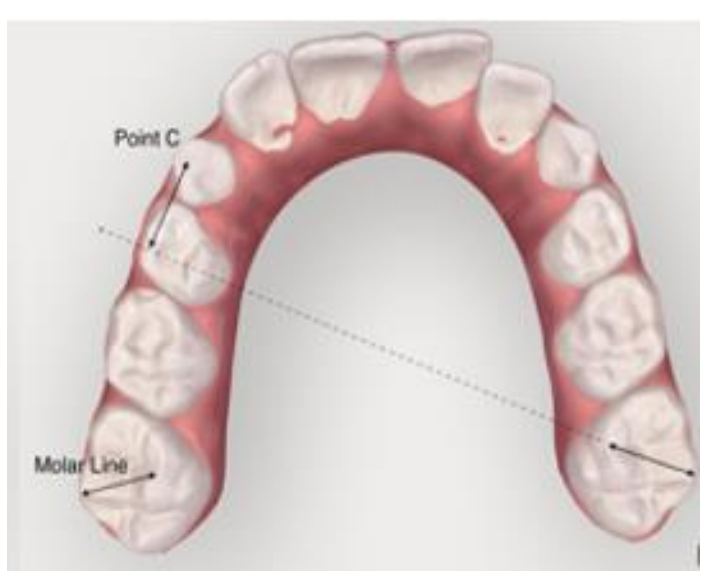

(b)

Figure 1. Ricketts'method to detect a mesiorotated upper first molar in (a) permanent dentition and (b) mixed dentition.

For each dental cast, the following variables were recorded and considered as potentially associated with UFM mesiopalatal rotation; gender, age, type of dentition (mixed or permanent), presence of 
anterior crowding, molar class relationship, and occurrence of diagnosed agenesis. Each patient was then included in one of the following anterior crowding categories assessed by using Little's index for maxillary arch (LIIMx) [23-25]; space excess in the upper arch (LIIMx $<0 \mathrm{~mm}$ ), no crowding $($ LIIMx $=0 \mathrm{~mm}$ ), light crowding (LIIMx = 1-3 mm), moderate crowding (LIIMx = 3-6 mm), or severe crowding (LIIMx $<7 \mathrm{~mm}$ ). The sample size was estimated starting from preliminary data of the present study, planning a minimum sample of 298 subjects in order to achieve an $80 \%$ power with an effect size of 0.2 . All the measures were performed by the same operator, and the method error was assessed by repeating the crowding category assessment 7 days after the first measurement on a randomly selected $20 \%$ of the whole sample. The concordance between the first and the second category assessment was $100 \%$.

Data were first reported as frequencies in the group of mixed/definitive dentition (number and percentage). Then, in order to assess any association between the presence of mesiorotated UFM and the occlusal variables, the Chi-square test was applied. An additional analysis of associations was also performed with calculation of the odds ratios (and confidence intervals). The analysis of data was performed with the software PSPP for Macintosh, version 1.2.0 and the significance level was set at 5\% $(p<0.05)$ for all statistical analyses.

\section{Results}

Descriptive statistics for the two groups are reported in Tables 1 and 2. There was no difference in the prevalence of UFM rotation between subjects with mixed or permanent dentition. In the whole sample $(n=349)$, there was a statistically significant association between mesiorotated UFM and anterior dental crowding (chi-square:10.59; $p=0.032$ ).

Table 1. Descriptive statistic (subject with mixed dentition) $(n=180)$.

\begin{tabular}{cc}
\hline & $\begin{array}{c}\text { Number of Subjects } \begin{array}{c}(\% \text { in the Group) with Mixed Dentition } \\
(\boldsymbol{n}=\mathbf{1 8 0})\end{array} \\
\text { Molar mesiorotation: } \\
\text { Correct position }\end{array}$ \\
Mesiorotated molar & $16(8.89 \%)$ \\
Occlusal relationship: & $164(91.11 \%)$ \\
Molar class I relationship & $52(28.89 \%)$ \\
End-to-end molar class & $78(43.33 \%)$ \\
Molar class II relationship & $15(8.33 \%)$ \\
Molar class III relationship & $35(19.44 \%)$ \\
Crowding/Diastema: & $7(3.89 \%)$ \\
Space excess in the dental arch & $19(10.56 \%)$ \\
No crowding & $42(23.33 \%)$ \\
Light crowding & $77(42.78 \%)$ \\
Moderate Crowding & $35(19.55 \%)$ \\
Severe crowding &
\end{tabular}

Table 2. Descriptive statistic (subject with permanent dentition) $(n=169)$.

\begin{tabular}{cc}
\hline & $\begin{array}{c}\text { Number of Subjects }\left(\begin{array}{c}\% \\
(\boldsymbol{n}=\mathbf{1 6 9 )}\end{array}\right. \\
\text { Molar mesionotation: } \\
\quad \text { Correct position } \\
\text { Mesionotated molar }\end{array}$ \\
Occlusal relationship: & $14(8.28 \%)$ \\
Molar class I relationship & $155(91.72 \%)$ \\
End-to-end molar class & $83(49.11 \%)$ \\
Molar class II relationship & $50(29.59 \%)$ \\
Molar class III relationship & $26(15.38 \%)$ \\
Crowding/Diastema: & $10(5.92 \%)$ \\
\hline
\end{tabular}


Table 2. Cont.

\begin{tabular}{cc}
\hline & $\begin{array}{c}\text { Number of Subjects (\% in the Group) with Permanent Dentition } \\
(\boldsymbol{n}=\mathbf{1 6 9 )}\end{array}$ \\
\hline Space excess in the dental arch & $10(5.92 \%)$ \\
No crowding & $19(11.24 \%)$ \\
Light crowding & $48(28.40 \%)$ \\
Moderate Crowding & $63(37.28 \%)$ \\
Severe crowding & $29(17.16 \%)$ \\
\hline
\end{tabular}

\subsection{Subjects with Mixed Dentition}

The occlusal variables of subjects with mixed dentition are described in Table 1 . Out of 180 subjects with mixed dentition, 164 showed UFM mesiopalatal rotation (91.11\%), where $85.36 \%$ (140 subjects) had anterior crowding. All patients with agenesis (6 subjects) showed UFM mesiopalatal rotation (100\% of cases). Stratifying the sample for Angle classes, UFM mesiopalatal rotation was present in $78.85 \%$ of Angle class I subjects, $97.44 \%$ of patients with class II occlusion, $91.43 \%$ of patients with class III, and in $100 \%$ of subjects with end-to-end relationship. The association between molar class and UFM rotation resulted statistically significant (Pearson's chi-square $=14.98 ; p=0.002$ ) (Table 3). Subjects with molar class II showed an OR $=10.2(95 \% \mathrm{CI}=2.16-48.22 ; p=0.0005)$ to present a mesiopalatal rotated UFM, compared with subjects with molar class I.

Table 3. Distribution of data for occlusal Angle class and presence of rotated UFM, with analyses of associations.

\begin{tabular}{ccccc}
\hline & \multicolumn{2}{c}{$\begin{array}{c}\text { Mixed Dentition }(n=180) \\
\text { Number of Subjects }(\% \text { on Group) }\end{array}$} & $\begin{array}{c}\text { Permanent Dentition }(n=169) \\
\text { Number of Subjects (\% on Group) }\end{array}$ \\
\hline Occlusal Class & Correct Position & Mesiopalatal Rotation & Correct Position & Mesiopalatal Rotation \\
\hline Molar class I & $11(21.15 \%)$ & $41(78.85 \%)$ & $6(7.23 \%)$ & $77(92.77 \%)$ \\
Molar class II & $2(2.56 \%)$ & $76(97.44 \%)$ & $4(8.0 \%)$ & $46(92 \%)$ \\
End-to-end & $0(0 \%)$ & $15(100 \%)$ & $3(11.54 \%)$ & $23(88.46 \%)$ \\
relationship & $3(8.57 \%)$ & $32(91.43 \%)$ & $1(10 \%)$ & $9(91.72 \%)$ \\
Molar class III & & $14.98(p=0.002)$ & & n.s. ${ }^{1}$ \\
Pearson chi-square & $15.24(p=0.002)$ & & n.s. ${ }^{1}$ \\
Likelihood Ratio & & $3.85(p=0.05)$ & n.s. ${ }^{1}$ \\
Linear-by-Linear & & 1 nonsignificant. &
\end{tabular}

\subsection{Subjects with Permanent Dentition}

The occlusal variables of subjects with permanent dentition are described in Table 2. Out of 169 subjects with permanent dentition, 155 subjects showed UFM mesiopalatal rotation (91.72\%), of which $90.32 \%$ (140 subjects) showed anterior crowding, while it was present only in 10 out of 14 patients with non-mesiorotated UFM (71.42\%). All patients with agenesis showed mesiorotated UFM (100\%). There was a statistically significant correlation between anterior dental crowding and UFM mesiorotation (Pearson's chi-square $=9.03 ; p=0.05$ ) (Table 4). Subjects with UFM mesiorotation showed an OR $=3.28(95 \% \mathrm{CI}=0.99-10.93 ; p=0.042)$ of having moderate/severe anterior dental crowding, compared to subjects without UFM mesiorotation. 
Table 4. Distribution of data for crowding and presence of rotated upper first molar (UFM), with corresponding analyses.

\begin{tabular}{|c|c|c|c|c|}
\hline \multirow[b]{2}{*}{ Crowding/Diastema } & \multicolumn{2}{|c|}{$\begin{array}{c}\text { Mixed Dentition }(n=180) \\
\text { Number of Subjects ( } \% \text { on Group) }\end{array}$} & \multicolumn{2}{|c|}{$\begin{array}{c}\text { Permanent Dentition ( } n=169) \\
\text { Number of Subjects ( } \% \text { on Group) }\end{array}$} \\
\hline & Correct Position & Mesiopalatal Rotation & Correct Position & Mesiopalatal Rotation \\
\hline No crowding & $3(15.79 \%)$ & $16(84.21 \%)$ & $4(21.05 \%)$ & $15(78.95 \%)$ \\
\hline Light crowding & $5(11.9 \%)$ & $37(88.1 \%)$ & $6(12.5 \%)$ & $42(87.5 \%)$ \\
\hline Moderate crowding & $6(7.79 \%)$ & $71(92.21 \%)$ & $4(6.35 \%$ & $59(93.65 \%)$ \\
\hline Severe crowding & $2(5.71 \%)$ & $33(94.29 \%)$ & $0(0 \%)$ & $29(100 \%)$ \\
\hline Space excess in the dental arch & $0(0 \%)$ & $7(100 \%)$ & $0(0 \%)$ & $10(100 \%)$ \\
\hline Pearson chi-square & \multicolumn{2}{|r|}{ n.s. } & \multicolumn{2}{|c|}{$9.03(p=0.05)$} \\
\hline Likelihood Ratio & \multicolumn{2}{|r|}{ n.s. } & \multicolumn{2}{|c|}{$11.03(p=0.026)$} \\
\hline Linear-by-Linear Association & \multicolumn{2}{|r|}{ n.s. } & \multicolumn{2}{|c|}{$8.46(p=0.004)$} \\
\hline
\end{tabular}

n.s. $=$ non-significant.

\section{Discussion}

Different studies identified the importance of UFM rotation in establishing a stable occlusion and its association with the molar class relationship especially in permanent dentition $[6,11,18,19]$. Furthermore, it was observed that UFM is able to influence the available space in the dental arch during orthodontic treatment [12-15,26]. Consequently, the present study investigated the prevalence of UFM mesiopalatal rotation and its association with anterior dental crowding and occlusal class in two groups of subjects with mixed and permanent dentition.

From the present data, the prevalence of UFM mesiopalatal rotation was high in both groups, in agreement with what is reported in previous studies $[6,8,9]$ that are based on subjects with permanent dentition. A low difference in prevalence between the two groups seems to suggest that the prevalence of UFM mesiopalatal rotation does not change significantly with the transition from mixed to permanent dentition. In subjects with permanent dentition, there was a statistically significant association between UFM mesiorotation and anterior dental crowding, and mesiorotated molar was present in $100 \%$ of subjects with severe crowding. Subjects with UFM mesiorotation showed an $\mathrm{OR}=3.28$ to show moderate/severe anterior dental crowding, compared to subjects without UFM mesiorotation. Thus, it could be hypothesized that a mesiorotated molar occlusal surface, occupying a larger space on the dental arch owing to its trapezoidal shape [11,12], reduces the available space in the dental arch, promoting the development of anterior crowding besides hindering proper dental intercuspation [5,12] and worsening the molar anteroposterior relationship [12].

Even if it is clinically renowned that the correction of UFM mesiorotation is able to increase the amount of available space during the orthodontic treatment [12-15,27], to the best of the authors' knowledge this is the first study that reports a direct association of UFM mesiorotation with the presence of anterior dental crowding. The orthodontic correction of UFM mesiopalatal rotation may provide between 1 and $2 \mathrm{~mm}$ of gain in arch perimeter [8].

In subjects with mixed dentition, a statistically significant association was observed with the occlusal class, differently to what was observed in permanent dentition. Subjects with molar class II showed an OR = 10.2 to present a mesiopalatal rotated UFM, compared to subjects with molar class I. Besides the present study, Lima et al. did not find any association between the severity of Class II and the magnitude of UFM rotation assessed by using the Ricketts` line method, in a group of 104 class II subjects with permanent dentition [1]. Nevertheless, the present investigation simply identified the presence/absence of mesiopalatal rotation without reporting the amount of molar rotation, thus is not directly comparable with what reported by Lima et al. [1].

As an association between UFM mesiorotation and anterior dental crowding was identified, it seems that subjects with mixed dentition and UFM mesiorotation should be considered at higher risk of having dental crowding.

The present study has some limitations, among them the absence of measure of the angle of UFM rotation that could allow in future studies an analysis of correlation of the amount UFM mesiorotation with anterior dental crowding. 


\section{Conclusions}

Based on the present methodology and the results obtained there seems to exist an association between UFM mesiopalatal rotation and anterior dental crowding in permanent dentition. Subjects with mixed dentition and UFM mesiorotation should be considered at higher risk of having dental crowding. Furthermore, in subjects with mixed dentition, an association between UFM rotation and class II malocclusion seems confirmed. Future studies should analyze the existence of a direct correlation between the amount of UFM rotation and the development of anterior upper dental crowding.

Author Contributions: Conceptualization, V.Q., A.N., and S.T.; methodology, A.N., S.T., and S.M.; validation, E.C., A.N., and S.T.; formal analysis, A.N. and S.T.; investigation, E.C.; resources, S.M., A.N., and S.T.; data curation, A.N., S.T., and C.E.; writing-original draft preparation, A.N., E.C., and S.T.; writing-review and editing, A.N. and S.T.; visualization, V.Q. and G.M.; supervision, V.Q. and G.M.; project administration, G.M. All authors have read and agreed to the published version of the manuscript.

Funding: This research received no external funding.

Acknowledgments: The authors acknowledge dr Floriana Bosco for her support in English language editing.

Conflicts of Interest: The authors declare no conflict of interest.

\section{References}

1. Lima, B.P.; Pinzan-Vercelino, C.R.M.; Dias, L.S.; Bramante, F.S.; Tavarez, R.R.D.J. Correlation between the Rotation of the First Molars and the Severity of Class II Division 1 Malocclusion. Sci. World J. 2015, 2015, 261485. [CrossRef] [PubMed]

2. Ferro, R.; Besostri, A.; Olivieri, A.; Quinzi, V.; Scibetta, D. Prevalence of cross-bite in a sample of Italian preschoolers. Eur. J. Paediatr. Dent. 2016, 17, 307-309. [PubMed]

3. Silvestrini-Biavati, A.; Migliorati, M.; Demarziani, E.; Tecco, S.; Silvestrini-Biavati, P.; Polimeni, A.; Saccucci, M. Clinical association between teeth malocclusions, wrong posture and ocular convergence disorders: An epidemiological investigation on primary school children. BMC Pediatr. 2013, 13, 12. [CrossRef] [PubMed]

4. Tecco, S.; Mummolo, S.; Marchetti, E.; Teté, S.; Campanella, V.; Gatto, R.; Gallusi, G.; Tagliabue, A.; Marzo, G. sEMG activity of masticatory, neck, and trunk muscles during the treatment of scoliosis with functional braces. A longitudinal controlled study. J Electromyogr. Kinesiol. 2011, 21, 885-892. [CrossRef] [PubMed]

5. Henry, R.G. Relationship of the maxillary first permanent molar in normal occlusion and malocclusion. Am. J. Orthod. 1956, 42, 288-306. [CrossRef]

6. Liu, D.; Melsen, B. Reappraisal of Class II molar relationships diagnosed from the lingual side. Clin. Orthod. Res. 2001, 4, 97-104.

7. Tecco, S.; Lacarbonara, M.; Dinoi, M.T.; Gallusi, G.; Marchetti, E.; Mummolo, S.; Campanella, V.; Marzo, G. The retrieval of unerupted teeth in pedodontics: Two case reports. J Med Case Rep. 2014, 8, 334. [CrossRef]

8. Giuntini, V.; Baccetti, T.; Defraia, E.; Cozza, P.; Franchi, L. Mesial rotation of upper first molars in Class II division 1 malocclusion in the mixed dentition: A controlled blind study. Prog. Orthod. 2011, 12, 107-113. [CrossRef]

9. Scanavini, P.E.; Jóias, R.P.; Vasconcelos, M.H.F.; Scanavini, M.A.; Paranhos, L.R. Assessment of first molars sagittal and rotational position in Class II, division 1 malocclusion. Dent. Press J. Orthod. 2013, 18, $106-111$. [CrossRef]

10. Lamons, F.; Holmes, C. The problem of the rotated maxillary first permanent molar. Am. J. Orthod. 1961, 47, 246-272. [CrossRef]

11. Dahlquist, A.; Gebauer, U.; Ingervall, B. The effect of a transpalatal arch for the correction of first molar rotation. Eur. J. Orthod. 1996, 18, 257-267. [CrossRef] [PubMed]

12. Braun, S.; Kusnoto, B.; Evans, C.A. The effect of maxillary first molar derotation on arch length. Am. J. Orthod. Dentofac. Orthop. 1997, 112, 538-544. [CrossRef]

13. Cooke, M.S.; Wreakes, G. Molar derotation with a modified palatal arch: An improved technique. Br. J. Orthod. 1978, 5, 201-203. [CrossRef]

14. Rebellato, J. Two-couple orthodontic appliance systems: Transpalatal arches. Semin. Orthod. 1995, 1, 44-54. [CrossRef] 
15. Rebellato, J. Two-couple orthodontic appliance systems: Activations in the transverse dimension. Semin. Orthod. 1995, 1, 37-43. [CrossRef]

16. Mummolo, S.; Nota, A.; De Felice, M.E.; Marcattili, D.; Tecco, S.; Marzo, G. Periodontal status of buccally and palatally impacted maxillary canines after surgical-orthodontic treatment with open technique. J. Oral Sci. 2018, 60, 552-556. [CrossRef]

17. Quinzi, V.; Caruso, S.; Mummolo, S.; Nota, A.; Angelone, A.M.; Mattei, A.; Gatto, R.; Marzo, G. Evaluation of Lower Dental Arch Crowding and Dimension after Treatment with Lip Bumper versus Schwarz Appliance. A Prospective Pilot Study. Dent. J. 2020, 8, 34.

18. Gündüz, E.; Zachrisson, B.U.; Hönigl, K.D.; Crismani, A.G.; Bantleon, H.P. An improved transpalatal bar design. Part I. Comparison of moments and forces delivered by two bar designs for symmetrical molar derotation. Angle Orthod. 2003, 73, 239-243.

19. Gündüz, E.; Crismani, A.G.; Bantleon, H.P.; Hönigl, K.D.; Zachrisson, B.U. An improved transpalatal bar design. Part II. Clinical upper molar derotation-case report. Angle Orthod. 2003, 73, 244-248.

20. Zachrisson, B.U. Important aspects of long-term stability. J. Clin. Orthod. 1997, 31, 562-583.

21. Tonni, I.; Iannazzi, A.; Piancino, M.G.; Costantinides, F.; Dalessandri, D.; Paganelli, C. Asymmetric molars' mesial rotation and mesialization in unilateral functional posterior crossbite and implications for interceptive treatment in the mixed dentition. Eur. J. Orthod. 2017, 39, 433-439. [CrossRef]

22. Ricketts, R.M. Occlusion-the medium of dentistry. J. Prosthet. Dent. 1969, 21, 39-60. [CrossRef]

23. Little, R.M. The irregularity index: A quantitative score of mandibular anterior alignment. Am. J. Orthod. 1975, 68, 554-563. [CrossRef]

24. Destang, D.L.; Kerr, W.J.S. Maxillary retention: Is longer better? Eur. J. Orthod. 2003, 25, 65-69. [CrossRef] [PubMed]

25. Quaglio, C.L.; de Freitas, K.M.S.; de Freitas, M.R.; Janson, G.; Henriques, J.F.C. Stability and relapse of maxillary anterior crowding treatment in class I and class II Division 1 malocclusions. Am. J. Orthod. Dentofac. Orthop. 2011, 139, 768-774. [CrossRef] [PubMed]

26. Mummolo, S.; Marchetti, E.; Albani, F.; Campanella, V.; Pugliese, F.; Di Martino, S.; Tecco, S.; Marzo, G. Comparison between rapid and slow palatal expansion: Evaluation of selected periodontal indices. Head Face Med. 2014, 10, 30. [CrossRef] [PubMed]

27. Orton, H.S. An evaluation of five methods of derotating upper molar teeth. Dent. Pract. Dent. Rec. 1966, 16, 279-286. [PubMed] 